\title{
Development of an intelligent adapter for metered dose inhalers
}

\author{
Mingrong Zhang ${ }^{1, a}$, Songhao Wang ${ }^{1}$ and Yu-Ching Yang ${ }^{1}$ \\ ${ }^{1}$ Mechanical Engineering Department, Kun Shan University, 71003 Tainan City, Taiwan
}

\begin{abstract}
To better coordinate the interaction of inhalation and aerosol release, an intelligent adapter (IA) was developed for metered dose inhalers (MDIs). The adapter included three main units: a signal acquisition device, a micro-control-unit (MCU), and an actuation mechanism. To fully study the effectiveness of the intelligent adapter, an inhalation simulation experiment was done, and two bands of MDI were used for the experiment. The results indicated that, when inhalation, the intelligent adapter can press down the MDI automatically; moreover, this intelligent adapter could achieve an aerosol-release time $\operatorname{Tr}$ of $0.4 \mathrm{~s}$ for MDI A and $0.60 \mathrm{~s}$ for MDI B, which compares very well with the existing pure mechanical systems at $0.8 \mathrm{~s}$ and $1.0 \mathrm{~s}$.
\end{abstract}

\section{Introduction}

The advantages of inhalation therapy includes direct impact on the respiratory airway, rapid onset of action, reduced dose and reduced adverse effects compared with injection and enteral therapies[1]. Furthermore, there are increasing potential applications for other diseases rather than only respiratory disease. For example, on June 29, 2014, the USA Food and Drug Administration (FDA) approved inhaled insulin for diabetes mellitus [2].

However, the inhalation technique is one of the most important factors that influences deposition of the inhaled drugs [3-5]. In the research of Piyush et al. [6], 300 bronchial asthma or COPD patients using different types of inhalation devices participated in an observational study. The maximum operation errors were observed in subjects using MDI (94.3\%) followed by DPI (82.3\%). For dry powder inhaler (DPI), a higher peak inspiratory flow rate is required, which prevents the application in children, the elderly and seriously ill patients $[1,7]$. Metered dose inhaler (MDI) is inspiratory flow independent, and coordination between inhalation and activation MDI is required [8]. However, MDI delivers larger dose (approximately $50 \%$ of the released drug) to the alveolar airways than DPI (approximately 30\%) does [9], and the coordination could be improved by using new technology.

This paper demonstrates an intelligent adapter, capable of auto-spray. Moreover, design considerations for coordination of the inhalation and the aerosol-release time is also discussed.

\section{Design and construction of an intelligent adapter}

The intelligent adapter consisted of three main units: a signal acquisition device, a micro-control-unit (MCU), and an actuation mechanism.

\section{1 Signal acquisition device}

The signal acquisition device (signal switch) was used to detect the action of inhalation. For the intelligent adapter, the signal switch included an impeller and a hall sensor as shown in figure 1 . The hall sensor was installed at the top of the device, and the magnet was installed at one of the leaves on the impeller. Because of gravity, the magnet was always at the bottom to prevent malfunctioning due to small vibrations. During inhalation, the airflow passes through the small hole into the impeller chamber and causes the impeller to rotate. When the magnet rotates to

\footnotetext{
a Corresponding author:153155269@qq.com
} 
the top, the hall sensor acquires the inhalation signal. Then, the rotation speed of the impeller was calibrated to the inhalation flow rate by the micro control unit.

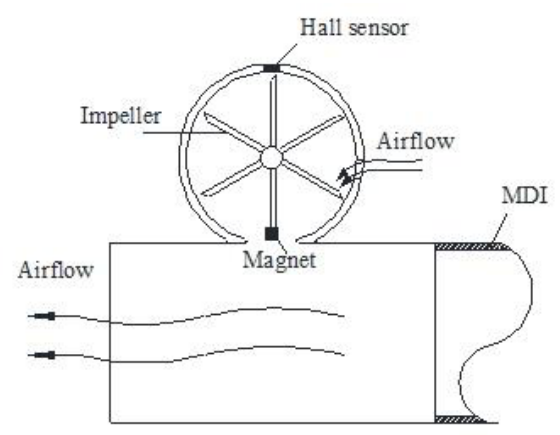

Figure 1. The structure of signal acquisition devices, an impeller and a hall sensor

\subsection{Micro-control-unit}

For controlling purposes, a micro-control-unit (MCU) (PIC 18F4550), with 32kbytes of program memory and a USB connection was used. The block diagram and printed-circuit-board of the control circuit are presented in figure 2 . It included a sensing circuit to calculate the inhalation process, a power supply circuit for the MCU and a motor to drive the actuation device. The UART(Universal Asynchronous Receiver/Transmitter) module was connected to a PC or laptop computer through the USB port.

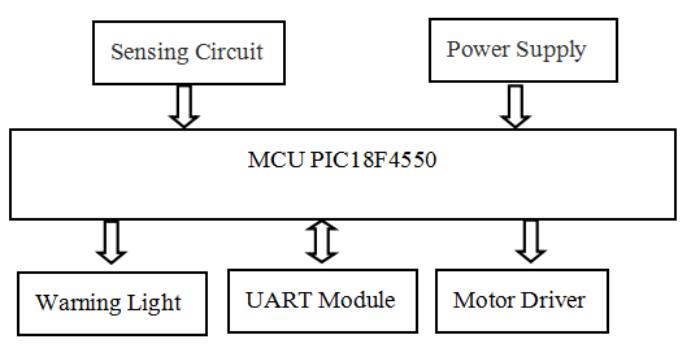

(a)

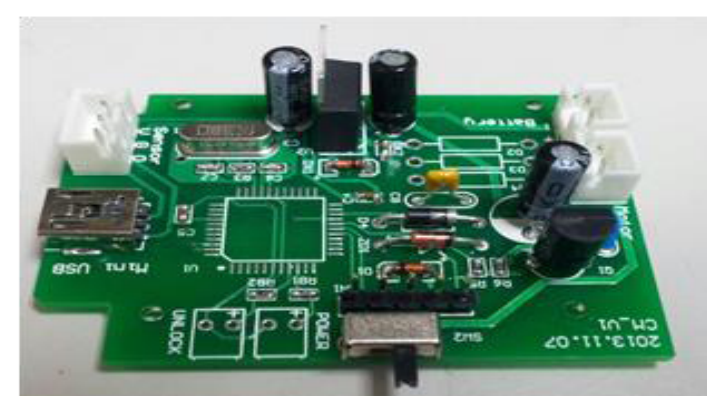

(b)

Figure 2. The block diagram (a) and PCB (b) of the control circuit

\subsection{Actuation mechanism}

A gear transmission mechanism was used to actuate the pressurized medicine canister because of its very high torque amplification efficiency. As shown in figure 3, three pairs of the gear were used to increase the torque from the small DC motor. In this study, the modulus of each gear was 0.6. The number of the teeth for each gear was: $\mathrm{g} 1=\mathrm{g} 3=\mathrm{g} 5=10$ and $\mathrm{g} 2=46, \mathrm{~g} 4=50, \mathrm{~g} 6=75$. Therefore, the torque could be amplified 172 times, and the mean push-down force could reach approximately $65 \mathrm{~N}$.
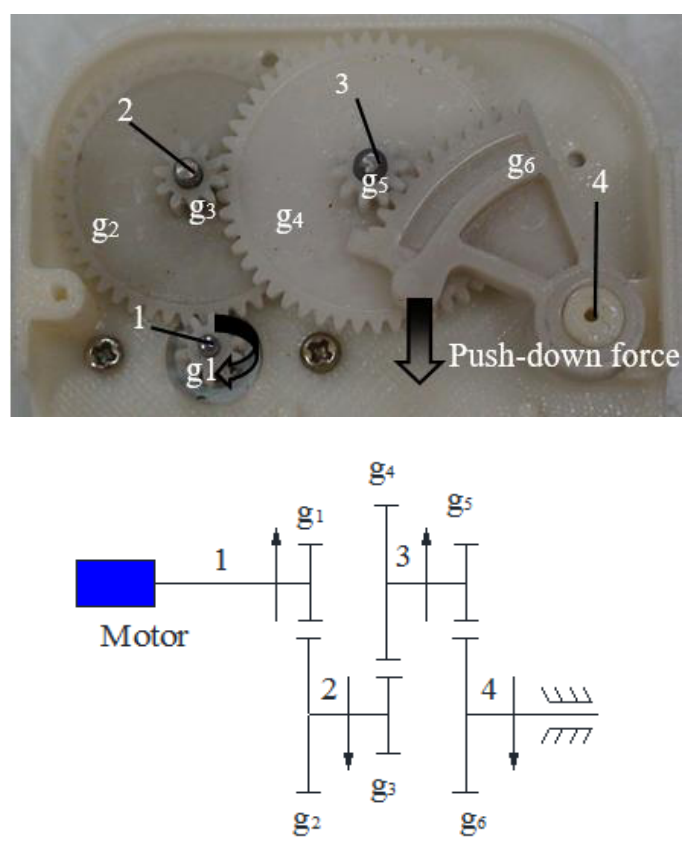

Figure 3. The gear transmission mechanism

\subsection{The key parametters of the intelligent adapter}

The feasibility for inhaled drugs depends mainly on aerosol release of MDI and the inhalation. The key technology of the intelligent adapter is to controll the aerosol-release time. For the intelligent adapter, the MDI was intalled on it. The aerosol-release time $\left(\mathrm{T}_{\mathrm{r}}\right)$ is divided into three periods: switch-on time $\left(\mathrm{T}_{\mathrm{s}}\right)$, mechatronics-hysteresis $\left(\mathrm{T}_{\mathrm{m}}\right)$ and intentional-delay $\left(\mathrm{T}_{\mathrm{i}}\right) . \mathrm{T}_{\mathrm{s}}$ is the period between the starting of inhalation and the connecting of the signal switch. For impeller and hall sensor signal switch, the inhalation flow rate that can just drive the impeller to rotate a whole circle is defined as switch-on flow-rate $\left(\mathrm{Q}_{\mathrm{S}}\right)$, the corresponding time is the switch-on time. $\mathrm{T}_{\mathrm{m}}$ is the period between signal switch on and the time when the aerosol starts to release when $T_{i}$ is 
equal to zero. $T_{i}$ is the time that can be adjusted by the computer programmng, and to meet a suitable $\mathrm{T}_{\mathrm{r}}$.

\section{Experiment}

The experiments were conducted to measure $\mathrm{T}_{\mathrm{r}}$. For the experiments, an inhalation simulator was designed to generate the inhalation waveforms by vacuum pump. A simulation upper respiratory system (as shown in figure 4) including mouth-throat, main bronchus and adapter intalled with MDI, was used to simulate the aerosol delivery. Additional camera system was used to record the aerosol deliver process and the movement of the adapter, then using the recording time to caculate $T_{r}$.

Two brands of MDI were selected for this study. MDI A delivers fenoterol at a single dose of $100 \mu \mathrm{g}$. CFC-free MDI B delivers salmeterol and fluticasone propionate single doses of $25 \mu \mathrm{g}$ and $250 \mu \mathrm{g}$, respectively.

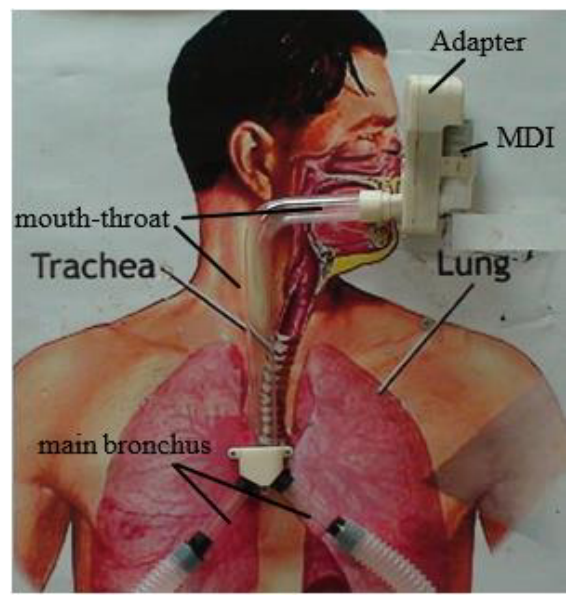

Figure 4. The upper respiratory system

\section{Results and Discussion}

Different signal acquisition devices have different switch-on time and switch-on flow-rate. Some autohalers with pure mechanical methods actuate the canister, however, the activating flow rate is above $30 \mathrm{~L} / \mathrm{min}$ [10]. With the help of modern mechatronics, the starting flow rate can be reduced to only $10 \mathrm{~L} / \mathrm{min}$. Ts for the autohaler is approximately $0.6 \mathrm{~s}$ compared with $0.2 \mathrm{~s}$ for the adapter.

Tm is related with the bands of the MDI. In this study, Tm are 0.2 and $0.4 \mathrm{~s}$ for MDI A and MDI B respectively. Assuming the autohaler had the same mechatronics -hysteresis as this study, $\operatorname{Tr}$ is shown in figure 5 . The intentional-delay is zero. The total aerosol-release time are $0.4 \mathrm{~s}$ or $0.6 \mathrm{~s}$ in this study and $0.8 \mathrm{~s}$ or $1.0 \mathrm{~s}$ for the autohaler for different bands of MDI. In the literature, the suggested aerosol-release times are $0-0.2 \mathrm{~s}$ [11] or $0.68 \mathrm{~s}$ for slow-deep inhalation [9]. Therefore, based on the results of this paper, it is better to start to actuate as soon as the system obtains the signal for some MDI. If $\operatorname{Tr}$ is small, it can be adjusted to a suitable value by the computer. Moreover, almost all the MDIs can use this intelligent adapter and it is reusable. The elddly, the child and the serious can operate it easily. However, for autohaler, the actor and the MDI are integrated and one-off; and the switch-on flow rate is higher than that of the intelligent adapter, it's not well for the weakness people.

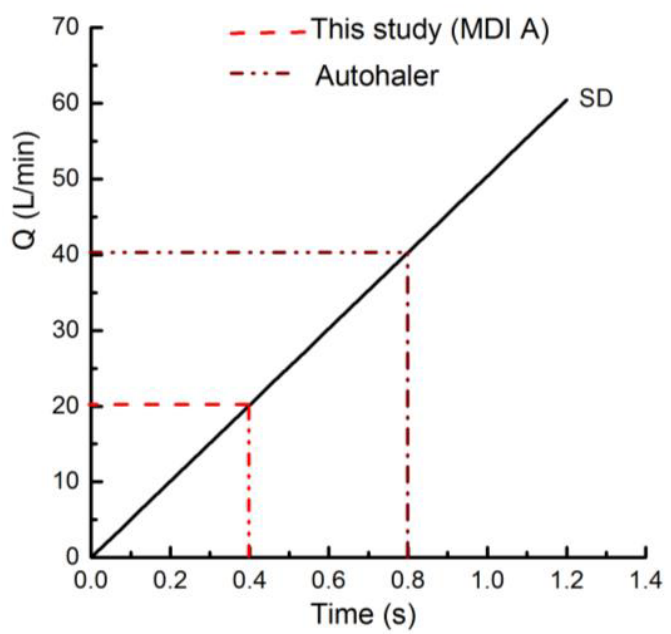

(a)

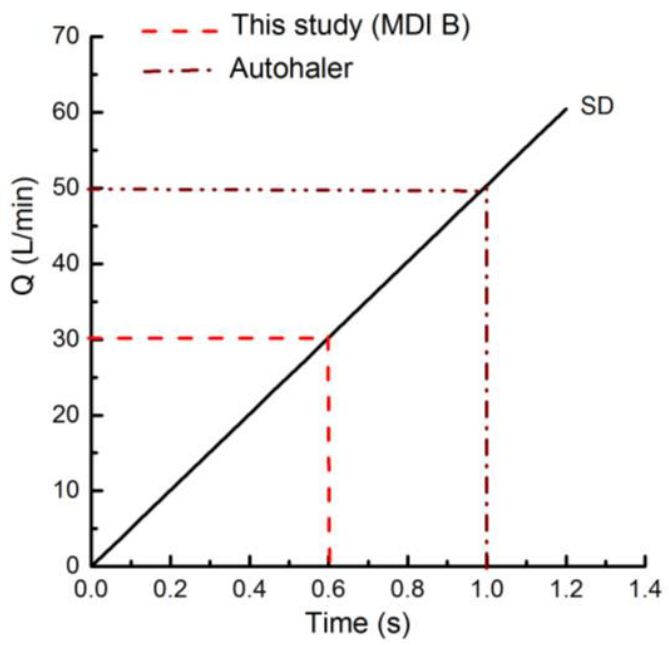

(b)

Figure 5. Comparison of aerosol-release time $T_{r}$ for MDI A (a) and MDI B (b) 


\section{Conclusion}

To help patients coordinate the interaction of inhalation and aerosol release, an intelligent metered dose inhaler has been developed. It is composed of an intelligent adapter and a metered dose inhaler. The added on mechatronic unit can detect the flow of inhalation and actuate the medicine canister at the specified release time.

The major advantages of the system are the low switch-on flow-rate $(10 \mathrm{~L} / \mathrm{min})$, short switch-on time, adjustable aerosol-release time, and low cost. Moreover, the feasibility of detecting and recording inhalation flow rates can help doctors monitor the process of treatment and train asthma or COPD patients to properly use MDI.

\section{References}

1. R. Dalby, J. Suman, ADV. DRUG DELIVER REV., 55, 779-791(2003)
2. J.J. McGrath, International Business Times, (2014)

3. G. M. Cochrane, CHEST, 117, 542-550 (2000)

4. D. Hochrainer, H. Hölz, C. Kreher, L. Scaffidi, M. Spallek, H. Wachtel, J Aerosol Med., 18, 273-282 (2005)

5. K. Nahar, N. Guta, R. Gauvin, S. Absar, EUR J PHARM, 49, 805-818 (2013)

6. P. Arora, L. Kumar, V. Vohra, R. Sarin, RESP MED ,108, 992-998 (2014)

7. H.W. Johannes, D.D. Nigel, M.W. Joyce, G.D. Sunalene, N.L. Peter, J PEDIATR, 135, 28-33 (1999)

8. J. Smith, J Aerosol Med Pulm Drug Deliv, 23, 25-37 (2010)

9. P. W. Longest, G. Tian, L.W. Ross, M. Hindle, Pharm Res, 29, 1670-1688 (2012)

10. D. Donnell, EUR REV MED PHARMACO, 5, 7-16 (2001)

11. M.E. Broeders, J. Molema, W.C. Hop, H.T. Folgering, J Aerosol Med, 16, 131-141(2003) 